\title{
ON A PROBLEM OF MAHLER FOR TRANSCENDENCY OF FUNCTION VALUES II
}

By

Kumiko NishioKa

\section{Introduction.}

In what follows, $\Omega$ is a $n \times n$ matrix whose entries are non-negative integers, and $\Omega$ satisfies:

(0) The characteristic polynomial of $\Omega$ is irreducible over $\boldsymbol{Q}$, the field of rational numbers, and $\Omega$ has the eigenvalues $\rho_{1}, \rho_{2}, \cdots, \rho_{n}$ such that $\rho_{1}>1$ and $\rho_{1}>\left|\rho_{2}\right| \geqq \cdots \geqq\left|\rho_{n}\right|$.

Let $\left(A_{i j}\right)$ be the classical adjoint (the transpose of the matrix of cofactors) of matrix $\Omega-\rho_{1} E$, where $E$ is the $n \times n$ identity matrix. For a non-negative integer $k$, we put $\Omega^{k}=\left(o_{i j}^{(k)}\right)$, and for a $n$-tuple of independent variables $z=\left(z_{1}, \cdots, z_{n}\right)$, we define

$$
T^{k} z=\left(z_{1}^{(k)}, \cdots, z_{n}^{(k)}\right), z_{i}^{(k)}=\Pi_{j=1}^{n} z_{j}^{{ }^{(k j}(k)} .
$$

Let $F$ be a finite algebraic number field and $f(z)=\sum_{h_{i} \geq 0} a_{h_{1} \cdots h_{n}} z_{1}{ }^{h_{1}} \cdots z_{n}{ }^{h_{n}}$ be a power series with coefficients in $F$. By $\overline{\boldsymbol{Q}}$ we denote the algebraic closure of $\boldsymbol{Q}$ in $\boldsymbol{C}$, the field of complex numbers. Mahler [4] proved:

THEOREM (Mahler). Let $f(z)$ be not algebraic over $\overline{\mathbf{Q}}\left(z_{1}, \cdots, z_{n}\right)$ and satisfy the functional equation

$$
f(T z)=\sum_{i=0}^{m} a_{i}(z) f(z)^{i} / \sum_{i=0}^{m} b_{i}(z) f(z)^{i},
$$

where the coefficients $a_{i}(z)$ and $b_{i}(z)$ are polynomials with algebraic coefficients and $m<\rho_{1} . \quad \Delta(z)$ denotes the resultant of $\sum_{i=0}^{m} a_{i}(z) u^{i}$ and $\sum_{i=0}^{m} b_{i}(z) u^{i}$ as polynomials in $u$. If $\alpha=\left(\alpha_{1}, \cdots, \alpha_{n}\right) \in \overline{\boldsymbol{Q}}^{n}$ satisfies that $\alpha_{1} \cdots \alpha_{n} \neq 0$, the real part of $\sum_{j=1}^{n}\left|A_{1 j}\right| \log \alpha_{j}$ is negative, $f(z)$ converges at $z=\alpha$ and $\Delta\left(T^{k} \alpha\right) \neq 0$ for all $k \geqq 0$, then $f(\alpha)$ is transcendental.

For example, $f(z)=\sum_{h=0}^{\infty} z^{2^{h}}$ satisfies the functional equation $f\left(z^{2}\right)=f(z)-z$. Then for an algebraic number such that $0<|\alpha|<1, f(\alpha)$ is transcendental. Refer to Loxton and van der Poorten [2], [3] for other examples. Mahler [5], [6] 
treated matrices of the form $\rho E$ and algebraic independency of values of several functions satisfying a certain type of functional equation. In [7], Mahler gave a summary of his earlier work and proposed three problems connected with it. Two of the three problems have been studied by Kubota, Loxton, van der Poorten and Masser. The present investigation is concerned with the remaining problem:

RROBLEM. Assume that $f(z)$ satisfies an algebraic functional equation of the form

$$
P(z, f(z), f(T z))=0,
$$

where $P(z, u, v) \neq 0$ is a polynomial in $u, v, z_{1}, \cdots, z_{n}$ with algebraic coefficients. To investigate the transcendency of function values $f(\alpha)$ where $\alpha$ is an algebraic point satisfying suitable further restrictions.

Our earlier paper [9] considered this problem in the case $n=1$. Now we consider the general case $n \geqq 1$, and treat more generalized power series and transformations. In [9], the coefficients of power series must satisfy some conditions but in this paper we shall show that the conditions are deduced from the functional equation.

\section{Preliminaries, theorems and lemmas.}

As usual, if $\alpha$ is an algebraic number, we denote by $|\alpha|$ the maximum of absolute values of the conjugates of $\alpha$ and by $d(\alpha)$ the least positive integer such that $d(\alpha) \alpha$ is an algebraic integer, and we set $\operatorname{size}(\alpha)=\max \{\log |\alpha|, \log d(\alpha)\}$. Assume that $\Omega$ satisfies $(0), t$ is a positive integer, and $\rho_{1} / t>1$. For a $n$-tuple of independent variables $z=\left(z_{1}, \cdots, z_{n}\right)$ and a non-negative integer $k$, we put

$$
T^{k} z=\left(z_{1}^{(k)}, \cdots, z_{n}^{(k)}\right), \quad z_{i}^{(k)}=\Pi_{j=1}^{n} z_{j}{ }^{{ }^{(k j}{ }^{(k)} / t^{k}} .
$$

Let $f(z)=\sum a_{h_{1} \cdots h_{n}} z_{1}{ }^{h_{1}} \cdots z_{n}{ }^{h_{n}}$ be a formal power series with powers being nonnegative rational numbers and coefficients in $F$. We may assume $a_{0 \ldots 0}=0$ without loss of generality. We consider the following four properties on $f(z)$.

(1) There are constants $c_{1}>0$ and $0 \leqq \eta<1 / n$ such that for any $h>0$ there exists a positive integer $\delta_{h} \leqq c_{1} h^{\eta}$ with $\delta_{h} h_{i} \in Z(1 \leqq i \leqq n)$ if $h_{i} \leqq h(1 \leqq i \leqq n)$ and $a_{h_{1} \ldots h_{n}} \neq 0$.

By the property (1), for a non-negative number $h$, the cardinality of terms $a_{h_{1} \cdots h_{n}} z_{1}^{h_{1}} \cdots z_{n}^{h_{n}}$ with $a_{h_{1} \cdots h_{n}} \neq 0$ and $h_{i} \leqq h(1 \leqq i \leqq n)$ is not greater than $\left(c_{1} h^{1+\eta}+1\right)^{n}$.

(2) $f(z)$ is not algebraic over $\overline{\mathbf{Q}}\left(z_{1}, \cdots, z_{n}\right)$. 
(3) $f(z)$ satisfies an algebraic functional equation of the form:

$$
Q_{0}(z, f(z)) f(T z)^{l}+Q_{1}(z, f(z)) f(T z)^{l-1}+\cdots+Q_{l}(z, f(z))=0,
$$

where $Q_{i}(z, u) \in \overline{\mathbf{Q}}\left[z_{1}, \cdots, z_{n}, u\right]$ and $Q_{0}(z, f(z)) \neq 0$.

Since we may assume that $Q_{0}(z, u), \cdots, Q_{l}(z, u)$ have no common divisor as polynomials in $u$, there are elements $g_{0}(z, u), \cdots, g_{l}(z, u)$ of $\overline{\mathbf{Q}}\left[z_{1}, \cdots, z_{n}, u\right]$ such that

$$
g(z)(\mathrm{say})=\sum_{i=0}^{l} g_{i}(z, u) Q_{i}(z, u)
$$

is independent of $u$ and not zero. We set

$$
m=\max _{0 \leqq i \leqq l} \operatorname{deg}_{u} Q_{i}(z, u) .
$$

(4) If $d_{h}$ is the least positive integer such that $d_{h} a_{h_{1} \cdots h_{n}}$ is an algebraic integer for all $\left(h_{1}, \cdots, h_{n}\right)$ with $h_{i} \leqq h(1 \leqq i \leqq n)$, then there are constants $c_{2}$ and $L \geqq 1$ such that

$$
\log \mid \overline{a_{h_{1} \cdots h_{n}}} \leqq c_{2}\left(\max \left\{h_{1}, \cdots, h_{n}\right\}\right)^{L}, \quad \log d_{h} \leqq c_{2} h^{L} .
$$

Let $\alpha=\left(\alpha_{1}, \cdots, \alpha_{n}\right) \in C^{n}, \alpha_{1} \cdots \alpha_{n} \neq 0$, and fix a branch of $\log \alpha_{i}(1 \leqq i \leqq n)$. For a non-negative integer $k$, we put

$$
\log \alpha_{i}^{(k)}=\sum_{j=1}^{n}\left(o_{i j}^{(k)} / t^{k}\right) \log \alpha_{j} .
$$

For a power series $f(z)$ with the property (1), we define

$$
f\left(T^{k} \alpha\right)=\sum a_{h_{1} \cdots h_{n}} e^{h_{1} \log \alpha_{1}^{(k)}+\cdots+h_{n} \log \alpha_{n}^{(k)},}
$$

if it absolutely converges.

THEOREM 1. Let $f(z)$ have the properties (1) (4). Let $\alpha=\left(\alpha_{1}, \cdots, \alpha_{n}\right) \in \overline{\mathbf{Q}}^{n}$, $\alpha_{1} \cdots \alpha_{n} \neq 0$, and suppose the real part of $\sum_{j=1}^{n}\left|A_{1 j}\right| \log \alpha_{j}$ is negative. Assume that $f\left(T^{k} \alpha\right)$ is defined and $g\left(T^{k} \alpha\right) \neq 0$ for any non-negative integer $k$. By $n_{0}$, we denote the rank of the multiplicative group generated by $\alpha_{1}, \cdots, \alpha_{n}$. If

$$
\begin{aligned}
& \left(\rho_{1} / t\right) \times \min \left\{\left(\rho_{1} / t\right)^{(1-n \eta) /(L+n(1+\eta)-1)},\left(\rho_{1} /\left|\rho_{2}\right|\right)^{(1-n \eta) / n(1+\eta)}\right\} \\
& >\left(t^{n} l\right)^{n+1} \times \max \left\{\rho_{1} / t, m\right\},
\end{aligned}
$$

then $f(\alpha)$ is transcendental.

THEOREM 2. If $f(z)$ satisfies (1) and (3), then $f(z)$ satisfies (4) with $L=$ $\max \{1+2 \eta+\varepsilon,(2+3 \eta)(n-1)\}$, where $\varepsilon$ is any positive number.

In the previous paper [9], we considered the transformation $T z=t_{p} z^{p}+\cdots+$ $t_{p+N} z^{p+N}$, where $t_{p}, \cdots, t_{p+N}$ are algebraic numbers, $p$ and $N$ are integers with 
$p \geqq 2$ and $N \geqq 0$, and $t_{p} t_{p+N} \neq 0$. In this case, we also have the following theorem.

THEOREM 3. Let $f(z)=\sum_{h=0}^{\infty} a_{h} z^{h}$ be a formal power series with powers being non-negative integers, the coefficients in $F$ and $a_{0}=0$. If $f(z)$ satisfies (3), then $f(z)$ satisfies (4) with $L=1+\varepsilon$, where $\varepsilon$ is an arbitrary positive number.

REMARK. Mahler considered the case $t=1, \eta=0$ and $l=1$, and the condition $m<\rho_{1}$ is needed. In this case, by Theorem 1 and Theorem 2 , we only need

$$
m<\rho_{1} \times \min \left\{\rho_{1}^{1 /(L+n-1)},\left(\rho_{1} /\left|\rho_{2}\right|\right)^{1 / n}\right\} .
$$

Note that the part of the minimum in the above inequality is greater than 1 .

EXAMPLE 1. Let $a$ be an integer greater than $1, \Omega=\left(\begin{array}{ll}a & 1 \\ 1 & 0\end{array}\right)$, and $t=1 . \Omega$ satisfies (0) and $\rho_{1}>a,\left|\rho_{2}\right|<1$. The power series

$$
f\left(z_{1}, z_{2}\right)=\Pi_{k=0}^{\infty}\left(1-z_{1}^{(k)} z_{2}^{(k)}\right)^{l k} \quad(l \geqq 1)
$$

satisfies the functional equation: $f(T z)^{l}\left(1-z_{1} z_{2}\right)=f\left(z_{1}, z_{2}\right)$. If $a_{1}=a_{2}=1$ and $a_{k}=a a_{k-1}+a_{k-2}$, then $z_{1}^{(k)} z_{2}^{(k)}=z_{1}{ }^{{ }}{ }_{k+1} z_{2}{ }^{a_{k}}$. It is shown that $f\left(z_{1}, z_{2}\right)$ is transcendental over $\overline{\boldsymbol{Q}}\left(z_{1}, z_{2}\right)$, and $f\left(z_{1}, z_{2}\right)$ satisfies (4) with $L=1$. By Theorem 1, if $l^{6} \leqq a, \alpha_{i} \in \overline{\mathbf{Q}}$ and $0<\left|\alpha_{i}\right|<1$, then $f\left(\alpha_{1}, \alpha_{2}\right)$ is transcendental.

EXAMPLE 2. Let $p$ be a positive integer, $t \geqq 2, p$ and $t$ be coprime and $p / t>1$. Assume that

$$
A(z, X)=a_{0}(z)+a_{1}(z) X+\cdots+a_{\iota}(z) X^{\imath} \in \overline{\mathbf{Q}}[z, x]
$$

satisfies $a_{0}(0)=0, a_{0}(z) \neq 0, a_{1}(0) \neq 0$, and the coefficients of $a_{i}(z)(0 \leqq i \leqq l)$ are positive. Put

$$
w_{0}(z)=0, \quad w_{n}(z)=A\left(z, w_{n-1}\left(z^{p / t}\right)\right) \quad(n \geqq 1) .
$$

Then $\operatorname{ord}_{z}\left(w_{n+1}(z)-w_{n}(z)\right) \geqq \operatorname{ord}_{z}\left(w_{n}\left(z^{p / t}\right)-w_{n-1}\left(z^{p / t}\right)\right)=(p / t) \operatorname{ord}_{z}\left(w_{n}(z)-w_{n-1}(z)\right)$. Since $\operatorname{ord}_{z}\left(w_{1}(z)-w_{0}(z)\right)>0$, there exists a formal power series $f(z)=\sum_{h=0}^{\infty} a_{h} z^{h}$ with the powers being non-negative rational numbers such that $\lim _{n \rightarrow \infty} w_{n}(z)=f(z)$. We have $f(z)=A\left(z, f\left(z^{p / t}\right)\right)$. If $a_{h} \neq 0$, then $h=n_{0}+n_{1}(p / t)+\cdots+n_{i}(p / t)^{i}$ for some non-negative integers $n_{0}, \cdots, n_{i}$. Therefore $f(z)$ satisfies (1) with $\eta=$ $\log t /(\log p-\log t)$. If $\operatorname{ord}_{z} a_{0}(z)=i_{0}$, then $a_{i_{0}(p / t) i} \neq 0$ for any $i \geqq 0$. Hence $f(z)$ is not a Puiseux series, and $f(z)$ is not algebraic over $\overline{\boldsymbol{Q}}(z)$. There is a constant $c>1$ computable from the coefficients of $A(z, X)$ such that

$$
\overline{\left|a_{h}\right|} \leqq c^{h}, \quad d_{h} \leqq c^{h} \quad(h>0) .
$$

By Theorem 1, if $(p / t)^{(1-\eta) /(1+\eta)}>(t l)^{2}, \alpha \in \overline{\boldsymbol{Q}}, 0<|\alpha|<1 / c$ and $a_{l}\left(\alpha^{(p / t)^{k}}\right) \neq 0$ for 
any $k \geqq 0$, then $f(\alpha)$ is transcendental. Especially, if $A(z, X)=z+X$ and $p>t^{5}$, then $f(z)=\sum_{h=0}^{\infty} z^{(p / t)^{h}}$ and $f(\alpha)$ is transcendental for any algebraic number such that $0<|\alpha|<1$. In the case $n>1$, we can also construct examples similarly.

We need some lemmas for the proof of theorems. Mahler [4] proved that if $\Omega$ satisfies (0), then $A_{11}, \cdots, A_{n_{1}}$ and also $A_{11}, \cdots, A_{1 n}$ are linearly independent over $\boldsymbol{Q}$, having the same sign, and $\Omega^{k}=\sum_{i=1}^{n} \rho_{i}{ }^{k} \Gamma_{i}(k \geqq 0)$ where $\Gamma_{i}$ is independent of $k$, the entries of $\Gamma_{1}$ are positive, and $\Gamma_{1}=A_{1}\left(A_{i 1} A_{1 j}\right)$ for some nonzero number $A_{1}$, these lead the following two lemmas.

LEMmA 1. Let $\alpha=\left(\alpha_{1}, \cdots, \alpha_{n}\right) \in C^{n}, \alpha_{1} \cdots \alpha_{n} \neq 0$, and $\Lambda=\sum_{j=1}^{n}\left|A_{1 j}\right| \log \alpha_{j}$. We denote the real part of $\Lambda$ by $\operatorname{Re} \Lambda$. Then for any $h_{1}, \cdots, h_{n} \in \boldsymbol{Q}$,

$$
\begin{aligned}
& \log \left|\left(\alpha_{1}^{(k)}\right)^{h_{1}} \cdots\left(\alpha_{n}^{(k)}\right)^{h_{n}}\right| \\
= & \left(\rho_{1} / t\right)^{k}\left|A_{1}\right|(\operatorname{Re} \Lambda) \sum_{i=1}^{n} h_{i}\left|A_{i 1}\right|+\phi\left(h_{1}, \cdots, h_{n}, k\right),
\end{aligned}
$$

where $\left|\phi\left(h_{1}, \cdots, h_{n}, k\right)\right| \leqq c_{3}\left(\sum_{i=1}^{n}\left|h_{i}\right|\right)\left(\left|\rho_{2}\right| / t\right)^{k}$ and $c_{3}$ depends only on $\Omega$ and $\alpha$.

LEMmA 2. Let $\alpha=\left(\alpha_{1}, \cdots, \alpha_{n}\right) \in \overline{\mathbf{Q}}^{n}, \alpha_{1} \cdots \alpha_{n} \neq 0$. Then there is a constant $c_{4}$ depending only on $\Omega$ and $\alpha$ such that $\log \left|\alpha_{i}^{(k)}\right| \leqq c_{4}\left(\rho_{1} / t\right)^{k}(1 \leqq i \leqq n, k \geqq 0)$, and there is a positive integer $d$ depending only on $\Omega$ and $\alpha$ such that $d^{\left[\left(\rho_{1} / t\right)^{k}\right]} \alpha_{i}^{(k)}$ $(1 \leqq i \leqq n, k \geqq 0)$ are algebraic integers.

LEMMA 3. Let $f(z)=\sum a_{h_{1} \cdots h_{n}} z_{1}{ }^{h_{1}} \cdots z_{n}{ }^{h_{n}}$ have the property (1). Assume that $a_{h_{1} \cdots h_{n}} \neq 0, \quad a_{h_{1}^{\prime} \cdots h_{n}^{\prime}} \neq 0, \quad\left(h_{1}, \cdots, h_{n}\right) \neq\left(h_{1}^{\prime}, \cdots, h_{n}^{\prime}\right)$ and $h_{i}, h_{i}^{\prime} \leqq h(1 \leqq i \leqq n)$. Then there is a positive constant $c_{5}$ depending only on $\Omega$ and $c_{1}$ such that

$$
\left|\sum_{i=1}^{n}\left(h_{i}-h_{i}^{\prime}\right)\right| A_{i 1}|| \geqq c_{5} h^{-n(1+\eta)+1} .
$$

Proof. Since $A_{11}, \cdots, A_{n 1}$ are linearly independent real numbers over $\boldsymbol{Q}$, $\left|A_{11}\right|, \cdots,\left|A_{n 1}\right|$ are also linearly independent over $\boldsymbol{Q}$. Since $\rho_{1}$ is an algebraic integer, $A_{i 1}(1 \leqq i \leqq n)$ are algebraic integers and $\left[\boldsymbol{Q}\left(A_{11}, \cdots, A_{n 1}\right): \boldsymbol{Q}\right] \leqq\left[\boldsymbol{Q}\left(\rho_{1}\right): \boldsymbol{Q}\right]$ $=n$. By the property (1), there is a positive integer $\delta_{h} \leqq c_{1} h^{\eta}$ such that $\delta_{h}\left(h_{i}-h_{i}^{\prime}\right) \in Z(1 \leqq i \leqq n)$. Therefore

$$
N_{Q\left(\rho_{i}\right) / Q} \delta_{h}\left(\sum_{i=1}^{n}\left(h_{i}-h_{i}^{\prime}\right)\left|A_{i 1}\right|\right) \geqq 1
$$

so we have Lemma 3,

LEMMA 4. Suppose that $B_{0} \neq 0, B_{1}, \cdots, B_{l}$ are algebraic numbers and $B_{0} \beta^{l}+B_{1} \beta^{l-1}+\cdots+B_{l}=0$. Then

$$
\left|\overline{B_{0} \beta}\right|<\left|\overline{B_{0}}\right|+\left|\overline{B_{1}}\right|+\cdots+\left|\overline{B_{l}}\right| .
$$

Futher, if $D$ is a positive integer such that $D B_{0}, D B_{1}, \cdots, D B_{l}$ are algebraic 
integers, then so is $D B_{0} \beta$.

\section{Proof of Theorem 1 .}

Let the power series $f(z)$ and the number $\alpha$ satisfy all the requirement of Theorem 1 and suppose, in addition, that $f(\alpha)$ is algebraic. Under these assumptions, we shall derive a contradiction, which proves the theorem. We set

$$
\Lambda=\sum_{j=1}^{n}\left|A_{1 j}\right| \log \alpha_{j} \text {, and } M=\max \left\{\rho_{1} / t, m\right\} .
$$

In the following, $c_{8}, c_{9}, \cdots$ denote constants greater than 1 depending on $\Omega, f, \alpha$ and the functional equation in (3), whose coefficients we may assume algebraic integers.

For any $r \geqq 0, f\left(T^{r} \alpha\right)$ and $f\left(T^{r+1} \alpha\right)$ are defined. Then by the property (3),

$$
\begin{aligned}
& Q_{0}\left(T^{r} \alpha, f\left(T^{r} \alpha\right)\right) f\left(T^{r+1} \alpha\right)^{l}+Q_{1}\left(T^{r} \alpha, f\left(T^{r} \alpha\right)\right) f\left(T^{r+1} \alpha\right)^{l-1} \\
& \quad+\cdots+Q_{\imath}\left(T^{r} \alpha, f\left(T^{r} \alpha\right)\right)=0 .
\end{aligned}
$$

Since $g\left(T^{r} \alpha\right) \neq 0$ by hypothesis, at least one of

$$
Q_{0}\left(T^{r} \alpha, f\left(T^{r} \alpha\right)\right), \cdots, Q_{l-1}\left(T^{r} \alpha, f\left(T^{r} \alpha\right)\right)
$$

is nonzero. We set

$$
j_{r}=\min \left\{j: Q_{j}\left(T^{r} \alpha, f\left(T^{r} \alpha\right)\right) \neq 0\right\},
$$

and define $Y_{r}(r \geqq 0)$ inductively, as follows :

$$
Y_{0}=1, \quad Y_{r}=Q_{j_{r-1}}\left(T^{r-1} \alpha, f\left(T^{r-1} \alpha\right)\right) Y_{r-1}{ }^{m} \quad(r \geqq 1) .
$$

Thus $Y_{r} \neq 0$ for all $r \geqq 0$. The next lemma gives estimates for these quantities.

LEMMA 5. For $r \geqq 1$,

$$
\left[F\left(\alpha_{1}, \cdots, \alpha_{n}, \cdots, \alpha_{1}^{(r)}, \cdots, \alpha_{n}^{(r)}, f(\alpha), \cdots, f\left(T^{r} \alpha\right): Q\right] \leqq c_{9}\left(l t^{n_{0}}\right)^{r},\right.
$$

and

$$
\operatorname{size}\left(Y_{r}\right), \quad \operatorname{size}\left(Y_{r} f\left(T^{r} \alpha\right)\right) \leqq c_{10} r M^{r}
$$

Proof. The first part of the lemma follows by induction using (3.1). Let $\operatorname{deg}_{z_{i}} Q_{j}(z, u)$ be not greater than $s$, the hause of $f(\alpha)$ and the coefficients of $Q_{j}(z, u)$ be not greater than $c_{8}$ with $c_{4}{ }^{n s} \leqq c_{8}$, and $D$ be a common multiple of $d(f(\alpha))$ and $d^{n s}\left(c_{4}\right.$ and $d$ are in Lemma 2). Then for any integer $r \geqq 1$, we can prove

$$
\left\{\begin{array}{l}
\left|\overline{Y_{r} \mid},\right| \overline{Y_{r} f\left(T^{r} \alpha\right)} \mid \leqq\left\{(l+1)(s+1)^{n}(m+1) c_{8}\right\}^{1+M+\cdots+M^{r-1}} c_{8}{ }^{r M^{r-1}+M^{r}} \\
D^{\left[r M^{r-1}+M^{r}\right]} Y_{r} \text { and } D^{\left[r M^{r-1}+M^{r}\right]} Y_{r} f\left(T^{r} \alpha\right) \text { are algebraic integers, }
\end{array}\right.
$$


by induction. If $r=1$, then $Y_{1}=Q_{j_{0}}(\alpha, f(\alpha))$,

$$
\left\{\begin{array}{l}
Y_{1} f(T \alpha)^{l-j_{0}}+\cdots+Q_{l}(\alpha, f(\alpha))=0, \\
\left|Q_{j}(\alpha, f(\alpha))\right| \leqq(s+1)^{n}(m+1) c_{8} \times c_{8}{ }^{1+m} \quad(0 \leqq j \leqq l), \\
D^{1+m} Q_{j}(\alpha, f(\alpha)) \quad(0 \leqq j \leqq l) \text { are algebraic integers. }
\end{array}\right.
$$

This implies (*) by Lemma 4, If $r>1$, then

$$
Y_{r} f\left(T^{r} \alpha\right)^{l-j_{r-1}}+\cdots+Y_{r-1}{ }^{m} Q_{l}\left(T^{r-1} \alpha, f\left(T^{r-1} \alpha\right)\right)=0 .
$$

By the induction hypothesis and Lemma 2, we obtain

$$
\left\{\begin{array}{l}
\left|Y_{r-1}{ }^{m} Q_{j}\left(T^{r-1} \alpha, f\left(T^{r-1} \alpha\right)\right)\right| \\
\leqq \\
\quad(s+1)^{n}(m+1) c_{8} c_{8}{ }^{\left(\rho_{1} / t\right) r-1} \\
\quad \times\left\{\left((l+1)(s+1)^{n}(m+1) c_{8}\right)^{1+M+\cdots+M^{r-2}} c_{8}{ }^{\left.(r-1) M^{r-2}+M^{r-1}\right\}^{m}},\right. \\
D^{\left[\left(\rho_{1} / t\right)^{r-1}\right]}\left(D^{\left[(r-1) M^{r-2}+M^{r-1}\right]}\right)^{m} Y_{r-1}{ }^{m} Q_{j}\left(T^{r-1} \alpha, f\left(T^{r-1} \alpha\right)\right) \\
\quad(0 \leqq j \leqq l) \text { are algebraic integers. }
\end{array}\right.
$$

This implies $(*)$ by Lemma 4 ,

By (2.2), we have

$$
\begin{aligned}
& \min \left\{\left(\rho_{1} / t\right)^{1 /(L+n(1+\eta)-1)},\left(\rho_{1} /\left|\rho_{2}\right|\right)^{1 / n(1+\eta)}\right\} \\
& >t^{n_{0}} l\left\{\left(t / \rho_{1}\right)\left(t^{n} l\right)^{n(1+\eta)} M\right\}^{\eta /(1+\eta)(1-n \eta)} \\
& \quad \times\left\{\left(t / \rho_{1}\right)\left(t^{n} l\right)^{n(1+\eta)} M\right\}^{1 /(1+\eta)(1-n \eta)} .
\end{aligned}
$$

Then there exists $q_{2}$ such that

$$
q_{2}>\left\{\left(t / \rho_{1}\right)\left(t^{n} 0 l\right)^{n(1+\eta)} M\right\}^{1 /(1+\eta)(1-n \eta)} \quad(\geqq 1)
$$

and

$$
\min \left\{\left(\rho_{1} / t\right)^{1 /(L+n(1+\eta)-1)},\left(\rho_{1} /\left|\rho_{2}\right|\right)^{1 / n(1+\eta)}\right\}>t^{n} 0 l q_{2}{ }^{1+\eta}
$$

By (3.3),

$$
q_{2}>\left(t / \rho_{1}\right) t^{n_{0}} l M\left(t^{n_{0}} l q_{2}^{\eta}\right)^{n(1+\eta)-1} .
$$

By (3.4) and (3.5), there is $q_{1}$ such that

$$
\begin{gathered}
q_{1}>t^{n_{0}} l q_{2}{ }^{\eta}, \\
q_{2}>\left(t / \rho_{1}\right) t^{n_{0}} l M{q_{1}}^{n(1+\eta)-1}, \\
\min \left\{\left(\rho_{1} / t\right)^{1 /(L+n(1+\eta)-1)},\left(\rho_{1} /\left|\rho_{2}\right|\right)^{1 / n(1+\eta)}\right\}>q_{1} q_{2} .
\end{gathered}
$$

By (3.7), $t^{n_{0}} l<q_{1} q_{2}$ so that by (3.8),

$$
t^{n} 0 l\left(q_{1} q_{2}\right)^{L}<\left(\rho_{1} / t\right) q_{1} q_{2} .
$$


The next lemma is one in relation to the construction of the auxiliary function.

LEMMA 6. Let $k$ be a positive integer and set $\gamma_{1}=2\left(c_{1}+1\right)^{n} q_{1}{ }^{n(1+\eta) k}$ and $\gamma_{2}=$ $q_{2}{ }^{(1+\eta) k}$. Then there are $\left[\gamma_{1}\right]+1$ polynomials $P_{j}(z)=\sum_{0 \leq h_{i} \leq\left[\gamma_{2}\right]} b_{h_{1} \cdots h_{n}}^{(j)} z_{1}{ }^{h_{1}} \cdots z_{n}{ }^{{ }_{n}}$ with degrees at most $\left[\gamma_{2}\right]$ whose coefficients are algebraic integers in $F$ with sizes at most $c_{11} k\left(q_{1} q_{2}\right)^{L k}$, such that the power series

$$
E_{k}(z)=\sum_{j=0}^{\left[\gamma_{1}\right]} P_{j}(z) f(z)^{j}=\sum b_{h_{1} \cdots h_{n}} z_{1}^{h_{1}} \cdots z_{n}{ }^{h_{n}}
$$

is not zero, but all the coefficients $b_{h_{1} \cdots h_{n}}$ with $h_{i}<\left(q_{1} q_{2}\right)^{k}(1 \leqq i \leqq n)$ vanish. Further,

and

$$
\operatorname{size}\left(b_{h_{1} \cdots h_{n}}\right) \leqq c_{12} k\left(\max \left\{h_{1}, \cdots, h_{n}\right\}\right)^{L},
$$

$$
\log \left|b_{h_{1} \cdots h_{n}}\right| \leqq c_{13} k\left(q_{1} q_{2}\right)^{L k}+c_{13} \max \left\{h_{1}, \cdots, h_{n}\right\}
$$

PROOF. Set $f(z)^{j}=\sum a_{h_{1} \cdots h_{n}}^{(j)} z_{1}{ }^{h_{1}} \cdots z_{n}{ }^{h_{n}}$, for $j \geqq 0$. By the properties (1) and (4), we have for $j \geqq 1$,

$$
\begin{aligned}
\left|\overline{a_{h}^{(j)} \cdots h_{n}}\right| & \leqq\left(c_{1}\left(\max \left\{h_{1}, \cdots, h_{n}\right\}\right)^{1+\eta}+1\right)^{n j} \times e^{c_{2}\left(n \times \max \left(h_{1}, \cdots, h_{n}\right) L\right.} \\
& \left.\leqq\left(\max \left\{h_{1}, \cdots, h_{n}\right\}\right)^{n(1+\eta) j} c_{16}{ }^{j+\left(\max \left(h_{1}, \cdots h_{n}\right)\right.}\right)^{L} .
\end{aligned}
$$

By the assumption that $f(\alpha)$ converges, $\log \left|a_{h_{1} \cdots h_{n}}\right| \leqq c_{17} \max \left\{h_{1}, \cdots, h_{n}\right\}$. Then for $j \geqq 1$,

$$
\left|a_{h}^{(j)} \cdots h_{n}\right| \leqq\left(\max \left\{h_{1}, \cdots, h_{n}\right\}\right)^{n(1+\eta) j} C_{18}^{j+\max \left\{h_{1}, \cdots, h_{n}\right\}} .
$$

The polynomials $P_{j}(z)$ have $\left(\left[\gamma_{1}\right]+1\right)\left(\left[\gamma_{2}\right]+1\right)^{n}$ coefficients $b_{h_{1}^{*} \cdot h_{n}}^{(j)}$ in all. We can achieve the property required of the auxiliary power series $E_{k}(z)$ by choosing $b_{h_{1} \cdots h_{n}}^{(j)}$ so as to satisfy the linear equations

$$
\sum a_{h_{1}^{\prime}-h_{1}, \cdots, h_{n}^{\prime}-h_{n}}^{(j)} b_{h_{1} \cdots h_{n}}^{(j)}=0 \quad\left(0 \leqq h_{i}^{\prime}<\left(q_{1} q_{2}\right)^{k}\right),
$$

where the sum is taken over all $h_{1}, \cdots, h_{n}, j$ satisfying $0 \leqq j \leqq\left[\gamma_{1}\right]$ and $0 \leqq h_{i} \leqq$ $\min \left\{\left[\gamma_{2}\right], h_{i}^{\prime}\right\}(1 \leqq i \leqq n)$. For any $b_{h_{1} \cdots h_{n}}^{(j)}, E_{k}(z)$ has the property (1) with the same $c_{1}$ and $\eta$ for $f(z)$. Therefore the number of linear equations is not greater than $\left(c_{1}\left(q_{1} q_{2}\right)^{(1+\eta) k}+1\right)^{n}$. The integer $D=\left(\Pi_{r=1}^{\left[\gamma_{1}\right]} d_{\left(q_{1} q_{2}\right) k / r}\right)^{n}$ will serve as a common denominator for all the $a_{h_{1} \cdots h_{n}}^{(j)}$ appering in those equations. The property (4) gives $\log D \leqq c_{19} k\left(q_{1} q_{2}\right)^{L k}$. By a standard version of Siegel's lemma, as given, for example, in Lang [1], page 4, the equations (3.10) have a non-trivial solution in which the $b_{h_{1} \cdots h_{n}}^{(j)}$ are algebraic integers in $F$ and

$$
\begin{aligned}
\operatorname{size}\left(b_{h_{1} \cdots h_{n}}^{(j)}\right) & \leqq c_{20} k q_{1}{ }^{n(1+\eta) k}+c_{21} k\left(q_{1} q_{2}\right)^{L k} \\
& \leqq c_{11} k\left(q_{1} q_{2}\right)^{L k} \quad\left(0 \leqq j \leqq\left[\gamma_{1}\right], 0 \leqq h_{i} \leqq\left[\gamma_{2}\right]\right)
\end{aligned}
$$

by (3.7). By the construction of $E_{k}(z)$, 
On a problem of Mahler for transcendency of function values II

$$
b_{h_{1}^{\prime} \cdots h_{n}^{\prime}}=\sum a_{h_{1}^{\prime}-h_{1}, \cdots, h_{n}^{\prime}-h_{n}}^{(j)} b_{h_{1} \cdots h_{n}}^{(j)}
$$

where the sum is taken over all $h_{1}, \cdots, h_{n}, j$ satisfying $0 \leqq j \leqq\left[\gamma_{1}\right]$ and $0 \leqq h_{i} \leqq$ $\min \left\{\left[\gamma_{2}\right], h_{1}^{\prime}\right\}(1 \leqq i \leqq n)$. In estimating $b_{h_{1}^{\prime} \cdots h_{n}^{\prime}}$ we can suppose that $\max \left\{h_{1}^{\prime}, \cdots, h_{n}^{\prime}\right\}$ $\geqq\left(q_{1} q_{2}\right)^{k}$, since otherwise $b_{h_{1}^{\prime} \cdots h_{n}^{\prime}}=0$. We have

$$
\begin{aligned}
\log \mid \overline{b_{h_{1}^{\prime} \cdots h_{n}^{\prime}} \mid} \leqq & \log \left(\left[\gamma_{1}\right]+1\right)\left(\left[\gamma_{2}\right]+1\right)^{n}+\left[\gamma_{1}\right] n(1+\eta) \log \max \left\{h_{1}^{\prime}, \cdots, h_{n}^{\prime}\right\} \\
& +\left(\left[\gamma_{1}\right]+\left(\max \left\{h_{1}^{\prime}, \cdots, h_{n}^{\prime}\right\}\right)^{L}\right) \log c_{16}+c_{11} k\left(q_{1} q_{2}\right)^{L k} \\
\leqq & c_{22} k\left(\max \left\{h_{1}^{\prime}, \cdots, h_{n}^{\prime}\right\}\right)^{L} .
\end{aligned}
$$

When $h^{\prime}=\max \left\{h_{1}^{\prime}, \cdots, h_{n}^{\prime}\right\}$, the integer $D_{h^{\prime}}=\left(\Pi_{r_{1}=1}^{\left[r_{1}\right]} d_{h^{\prime} / r}\right)^{n}$ will serve as a common denominator for all the $a_{h_{1}^{\prime}-h_{1}, \cdots, h_{n}^{\prime}-h_{n}}^{(j)}$ appearing in (3.11), so that

$$
\log d\left(b_{h_{1}^{\prime} \cdots h_{n}^{\prime}}\right) \leqq \log D_{h^{\prime}} \leqq c_{23} k h^{\prime L} .
$$

Finally, again using (3.11),

$$
\begin{aligned}
\log \left|b_{h_{1}^{\prime} \cdots h_{n}^{\prime}}\right| \leqq & \log \left(\left[\gamma_{1}\right]+1\right)\left(\left[\gamma_{2}\right]+1\right)^{n}+n(1+\eta)\left[\gamma_{1}\right] \log \max \left\{h_{1}^{\prime} ; \cdots, h_{n}^{\prime}\right\} \\
& +\left(\left[\gamma_{1}\right]+\max \left\{h_{1}^{\prime}, \cdots, h_{n}^{\prime}\right\}\right) \log c_{18}+c_{11} k\left(q_{1} q_{2}\right)^{L k} \\
\leqq & c_{13} k\left(q_{1} q_{2}\right)^{L k}+c_{14} \max \left\{h_{1}^{\prime}, \cdots, h_{n}^{\prime}\right\} .
\end{aligned}
$$

This completes the proof of lemma.

Let $E_{k}(z)$ be the function constructed in Lemma 6, We set

$$
H=\min \left\{\sum_{i=1}^{n} h_{i}\left|A_{i 1}\right|: b_{h_{1} \cdots h_{n}} \neq 0\right\},
$$

and $H=\sum_{i=1}^{n} H_{i}\left|A_{i 1}\right|$. Let $K$ be the integer such that $\left(q_{1} q_{2}\right)^{K} \leqq \max \left\{H_{1}, \cdots, H_{n}\right\}$ $<\left(q_{1} q_{2}\right)^{K+1}$. By Lemma 6, we have $\max \left\{H_{1}, \cdots, H_{n}\right\} \geqq\left(q_{1} q_{2}\right)^{k}$, so that $K \geqq k$.

LEMMA 7. For $k \geqq 1$, we have

$$
\begin{aligned}
& {\left[\boldsymbol{Q}\left(Y_{K^{[}}^{\left[\gamma_{1}\right]} E_{k}\left(T^{K} \alpha\right)\right): \mathbf{Q}\right] \leqq c_{24}\left(t^{n} 0 l\right)^{K},} \\
& \operatorname{size}\left(Y_{K}{ }^{\left[\gamma_{1}\right]} E_{k}\left(T^{K} \alpha\right)\right) \leqq c_{25} K\left(q_{1} q_{2}\right)^{L K}+c_{26}\left(\left(\rho_{1} / t\right) q_{2}{ }^{1+\eta}\right)^{K}+c_{27} K\left(q_{1}{ }^{n(1+\eta)} M\right)^{K} .
\end{aligned}
$$

PROOF. The first assertion follows at once from Lemma 5, For the second, we use the representation

$$
Y_{K}^{\left[\gamma_{1}\right]} E_{k}\left(T^{K} \alpha\right)=\sum_{j=0}^{\left[\gamma_{1}\right]} P_{j}\left(T^{K} \alpha\right)\left(Y_{K} f\left(T^{K} \alpha\right)\right)^{j} Y_{K^{\left[\gamma_{1}\right]-j}}
$$

From Lemma 2, 5 and the estimate for the size of coefficients of the polynomials $P_{j}(z)$ in Lemma 6, we find

$$
\begin{aligned}
\operatorname{size}\left(Y_{K}{ }^{\left[\gamma_{1}\right]} E_{k}\left(T^{K} \alpha\right)\right) \leqq & \log \left(\left[\gamma_{1}\right]+1\right)\left(\left[\gamma_{2}\right]+1\right)^{n}+c_{11} k\left(q_{1} q_{2}\right)^{L k} \\
& +c_{28} n\left[\gamma_{2}\right]\left(\rho_{1} / t\right)^{K}+\left[\gamma_{1}\right] c_{10} K M^{K} .
\end{aligned}
$$


This yields the assertion of the lemma.

LEMMA 8. If $k$ is sufficiently large, then $Y_{K}^{\left[\gamma_{1}\right]} E_{k}\left(T^{K} \alpha\right)$ is not zero and

$$
\log \left|Y_{K^{K}}^{\left[r_{1}\right]} E_{k}\left(T^{K} \alpha\right)\right| \leqq(\operatorname{Re} \Lambda / 2)\left|A_{1}\right|\left(\min _{1 \leq i \leq n}\left|A_{i 1}\right|\right)\left(\rho_{1} / t\right)^{K}\left(q_{1} q_{2}\right)^{K}
$$

PROOF. We can write

$$
E_{k}(z)=b_{H_{1} \cdots H_{n}} z_{1}{ }^{H_{1}} \cdots z_{n}{ }^{H_{n}}\left\{1+\sum\left(b_{h_{1} \cdots h_{n}} / b_{H_{1} \cdots H_{n}}\right) z_{1}{ }^{h_{1}-H_{1}} \cdots z_{n}{ }^{h_{n}-H_{n}}\right\},
$$

where the sum is taken over all $\left(h_{1}, \cdots, h_{n}\right)$ such that $\sum_{i=1}^{n} H_{i}\left|A_{i 1}\right|<\sum_{i=1}^{n} h_{i}\left|A_{i 1}\right|$. By using the fundamental inequality of transcendence theory (If $\beta$ is a nonzero algebraic number, then $\log |\beta| \geqq-2[\boldsymbol{Q}(\beta): \boldsymbol{Q}] \operatorname{size}(\beta)$.), and Lemma 6 ,

$$
\begin{aligned}
& \log \left|b_{h_{1} \cdots h_{n}} / b_{H_{1} \cdots H_{n}}\right| \\
\leqq & c_{13} k\left(q_{1} q_{2}\right)^{L k}+c_{13} \max \left\{h_{1}, \cdots, h_{n}\right\}+c_{29} k\left(\max \left\{H_{1}, \cdots, H_{n}\right\}\right)^{L} \\
\leqq & c_{30} K\left(q_{1} q_{2}\right)^{L K}+c_{31} \sum_{i=1}^{n} h_{i}\left|A_{i 1}\right| .
\end{aligned}
$$

For any nonnegative integer $y$, we set

$$
B_{y}=\sum\left(b_{h_{1} \cdots h_{n}} / b_{H_{1} \cdots H_{n}}\right) e^{\left(h_{1}-H_{1}\right) \log \alpha_{1}^{(k)}+\cdots+\left(h_{n}-H_{n}\right) \log \alpha_{n}^{(k)},}
$$

where the sum is taken over all $\left(h_{1}, \cdots, h_{n}\right)$ satisfying

$$
\sum_{i=1}^{n} H_{i}\left|A_{i 1}\right|+y+1 \geqq \sum_{i=1}^{n} h_{i}\left|A_{i 1}\right|>\sum_{i=1}^{n} H_{i}\left|A_{i 1}\right|+y \text {. }
$$

Then

$$
E_{k}\left(T^{K} \alpha\right)=b_{H_{1} \cdots H_{n}} e^{H_{1} \log \alpha_{1}^{(k)}+\cdots+H_{n} \log \alpha_{n}^{(k)}}\left(1+\sum_{y=0}^{\infty} B_{y}\right) .
$$

Using the fact that $E_{k}(z)$ has the property (1) with the same $c_{1}$ and $\eta$ for $f(z)$, Lemma 1 and (3.12), we have

$$
\begin{aligned}
\log \left|B_{y}\right| \leqq & \log c_{32}\left(\left(q_{1} q_{2}\right)^{K+1}+y+1\right)^{n(1+\eta)} \\
& +c_{30} K\left(q_{1} q_{2}\right)^{L K}+c_{31} \max \left\{\sum_{i=1}^{n} h_{i}\left|A_{i 1}\right|\right\} \\
& +\left(\rho_{1} / t\right)^{K}\left|A_{1}\right|(\operatorname{Re} \Lambda) \min \left\{\sum_{i=1}^{n}\left(h_{i}-H_{i}\right)\left|A_{i 1}\right|\right\} \\
& +c_{3} \max \left\{\sum_{i=1}^{n}\left|h_{i}-H_{i}\right|\right\}\left(\left|\rho_{2}\right| / t\right)^{K},
\end{aligned}
$$

where $\max$ and $\min$ are taken over all $\left(h_{1}, \cdots, h_{n}\right)$ satisfying $(* *)$. If $y \geqq 1$, then $\min \left\{\sum_{i=1}^{n}\left(h_{i}-H_{i}\right)\left|A_{i 1}\right|\right\}>y \geqq 1$. By (3.8), if $k$ is sufficiently large, then

$$
\log \left|B_{y}\right| \leqq(\operatorname{Re} \Lambda / 2)\left|A_{1}\right|\left(\rho_{1} / t\right)^{K} y
$$

for any $y \geqq 1$. If $y=0$, then by Lemma 3 , for any $\left(h_{1}, \cdots, h_{n}\right)$ satisfying $(* *)$,

$$
\sum_{i=1}^{n}\left(h_{i}-H_{i}\right)\left|A_{i 1}\right| \geqq c_{33}{ }^{-1}\left(q_{1} q_{1}\right)^{(-n(1+\eta)+1) K} .
$$

By this inequality and (3.8), 


$$
\log \left|B_{0}\right| \leqq(\operatorname{Re} \Lambda / 2)\left|A_{1}\right|\left(\rho_{1} / t\right)^{K} c_{33}{ }^{-1}\left(q_{1} q_{2}\right)^{(-n(1+\eta)+1) K},
$$

if $k$ is sufficiently large. By (3.14) and (3.15), we have

$$
\left|\sum_{y=0}^{\infty} B_{y}\right|<1 \text {, }
$$

if $k$ is sufficiently large. Therefore, by (3.13), $E_{k}\left(T^{K} \alpha\right)$ is not zero, if $k$ is sufficiently large. By Lemma 1, 5, 6, (3.7) and (3.8), we have

$$
\begin{aligned}
& \log \left|Y_{K}{ }^{\left[\gamma_{1}\right]} E_{k}\left(T^{K} \alpha\right)\right| \\
\leqq & 2\left(c_{1}+1\right)^{n} q_{1}{ }^{n(1+\eta) k} c_{10} K M^{K}+c_{13} k\left(q_{1} q_{2}\right)^{L k}+c_{13} \max \left\{H_{1}, \cdots, H_{n}\right\} \\
& +\left(\rho_{1} / t\right)^{K}\left|A_{1}\right|(\operatorname{Re} \Lambda) \sum_{i=1}^{n} H_{i}\left|A_{i 1}\right|+c_{3}\left(\sum_{i=1}^{n} H_{i}\right)\left(\left|\rho_{2}\right| / t\right)^{K}+\log 2 . \\
\leqq & (\operatorname{Re} \Lambda / 2)\left|A_{1}\right|\left(\min _{1 \leq i \leq n}\left|A_{i 1}\right|\right)\left(\rho_{1} / t\right)^{K}\left(q_{1} q_{2}\right)^{K},
\end{aligned}
$$

if $k$ is sufficiently large.

To complete the proof of Theorem 1, we apply the fundamental inequality of transcendence theory to the number $Y_{K}{ }^{\left[\gamma_{1}\right]} E_{k}\left(T^{K} \alpha\right)$. By Lemma 7 and Lemma 8, we obtain

$$
\begin{aligned}
& (\operatorname{Re} \Lambda / 2)\left|A_{1}\right|\left(\min _{1 \leq i \leq n}\left|A_{i 1}\right|\right)\left(\rho_{1} / t\right)^{K}\left(q_{1} q_{2}\right)^{K} \\
\geqq & -2 c_{24}\left(t^{n} l\right)^{K}\left\{c_{25} K\left(q_{1} q_{2}\right)^{L K}+c_{26}\left(\left(\rho_{1} / t\right) q_{2}{ }^{1+\eta}\right)^{K}+c_{27} K\left(q_{1}{ }^{n(1+\eta)} M\right)^{K}\right\},
\end{aligned}
$$

providing $k$ is sufficiently large. Since $\operatorname{Re} A<0$ and $K \geqq k$, this contradicts (3.6), (3.7) and (3.9).

\section{Proof of Theorem 2.}

At the first, we prove the theorem in the case where $f(z)$ is a power series with powers being non-negative integers and $t=1$. Adopting $\Omega^{r}$ for $\Omega$, if necessary, we may assume that the entries of $\Omega$ are greater than 1 . Let

$$
S=\left\{\left(\lambda_{1}, \cdots, \lambda_{n}\right): 0 \leqq \lambda_{i} \in Z\right\},
$$

and we define $\left(\lambda_{1}, \cdots, \lambda_{n}\right)<\left(\lambda_{1}^{\prime}, \cdots, \lambda_{n}^{\prime}\right)$ if and only if $\lambda_{1}+\cdots+\lambda_{n}<\lambda_{1}^{\prime}+\cdots+\lambda_{n}^{\prime}$ or $\lambda_{1}+\cdots+\lambda_{n}=\lambda_{1}^{\prime}+\cdots+\lambda_{n}^{\prime}$ and $\lambda_{1}=\lambda_{1}^{\prime}, \cdots, \lambda_{i}=\lambda_{i}^{\prime}, \lambda_{i+1}<\lambda_{i+1}^{\prime}$. Then $S$ is a totally ordered set. For $\lambda=\left(\lambda_{1}, \cdots, \lambda_{n}\right) \in S$ and $z=\left(z_{1}, \cdots, z_{n}\right)$, we set $|\lambda|=\lambda_{1}+\cdots+\lambda_{n}$, $\lambda !=\lambda_{1} ! \cdots \lambda_{n} !, z^{\lambda}=z_{1}^{\lambda_{1}} \cdots z_{n}^{\lambda_{n}}, \frac{\partial^{\lambda}}{\partial z^{\lambda}}=\frac{\partial^{\lambda_{1}}}{\partial z_{1}^{\lambda_{1}}} \cdots \frac{\partial^{\lambda_{n}}}{\partial z_{n}^{\lambda_{n}}}$. Then $f(z)=\sum_{\lambda \in S} a_{\lambda} z^{\lambda}$. By the property (3), there is a polynomial $P(z, u, v) \in \bar{Q}\left[z_{1}, \cdots, z_{n}, u, v\right]$ with coefficients being algebraic integers such that

$$
\begin{aligned}
& P(z, f(z), f(T z))=0, \\
& P_{u}(z, f(z), f(T z))=\text { (put) } \sum_{\lambda \in S} A_{\lambda} z^{\lambda} \neq 0,
\end{aligned}
$$


where $P_{u}(z, u, v)$ is the partial derivative of $P(z, u, v)$ in $u$. We denote by $\lambda_{0}$, the least $\lambda$ with $A_{\lambda} \neq 0$, and put $\left|\lambda_{0}\right|=m_{0}$. Let $\left\{y_{\lambda}\right\}_{\lambda \in S}$ be variables, and we set

$$
\begin{aligned}
& f(y, z)=\sum_{\lambda \in S} y_{\lambda} z^{\lambda}, \\
& P_{u}(z, f(y, z), f(y, T z))=\sum_{\lambda \in S} A_{\lambda}(y) z^{\lambda}, \\
& P(z, f(y, z), f(y, T z))=\sum_{\lambda \in S} B_{\lambda}(y) z^{\lambda} .
\end{aligned}
$$

If $\lambda \in Z^{n}$ and $\notin S$, then we put $A_{\lambda}(y)=B_{\lambda}(y)=0$. Hence $A_{\lambda}(y)$ and $B_{\lambda}(y)$ are polynomials in $\left\{y_{\lambda}\right\}_{\lambda \in S}$ with coefficients being algebraic integers. Substituting $a_{\lambda}$ to $y_{\lambda}$ in $A_{\mu}(y)$ and $B_{\mu}(y)$, we obtain $A_{\mu}$ and 0 respectively.

LEMMA 9. Let $\nu, \mu \in S$, and $2|\nu|>|\mu|$. Then $\operatorname{deg}_{y_{\nu}} B_{\mu}(y) \leqq 1$ and the coefficient of $y_{\nu}$ in $B_{\mu}(y)$ is $A_{\mu-\nu}(y)$.

PROOF. Since $2|\nu|>|\mu|, \operatorname{deg}_{y_{\nu}} B_{\mu} \leqq 1$. The coefficient of $y_{\nu}$ in $B_{\mu}(y)$ is equal to

$$
\begin{aligned}
& \left.\frac{\partial}{\partial y_{\nu}} \cdot \frac{1}{\mu !} \cdot \frac{\partial^{\mu}}{\partial z^{\mu}} P(z, f(y, z), f(y, T z))\right|_{z=0} \\
= & \left.\frac{\partial}{\partial y_{\nu}} \cdot \frac{1}{\mu !} \cdot \frac{\partial^{\mu}}{\partial z^{\mu}} P\left(z, \sum_{\lambda s_{\mu}} y_{\lambda} z^{\lambda}, \sum_{\lambda \Omega_{s} \mu} y_{\lambda} z^{\lambda \Omega}\right)\right|_{z=0}
\end{aligned}
$$

Since $2|\nu|>|\mu|, y_{\nu}$ does not appear in $\sum_{\lambda \Omega \leq \mu} y_{\lambda} z^{\lambda \Omega}$ and therefore (4.1) is equal to

$$
\begin{aligned}
& \left.\frac{1}{\mu !} \cdot \frac{\partial^{\mu}}{\partial z^{\mu}}\left\{P_{u}\left(z, \sum_{\lambda \leq \mu} y_{\lambda} z^{\lambda}, \sum_{\lambda \Omega_{\leq} \mu} y_{\lambda} z^{\lambda \Omega}\right) z^{\nu}\right\}\right|_{z=0} \\
= & \left.\frac{1}{\mu !} \cdot \frac{\partial^{\mu}}{\partial z^{\mu}}\left\{P_{u}(z, f(y, z), f(y, T z)) z^{\nu}\right\}\right|_{z=0} \\
= & A_{\mu-\nu}(y) .
\end{aligned}
$$

Let $P(z, u, v)=\sum_{i \in S} \sum_{j=0}^{J} \sum_{k=0}^{K} b_{i j k} z^{i} u^{j} v^{k}$, where $b_{i j k}$ are algebraic integers. We set

$$
M=\max \left\{\sum_{i \in S} \sum_{j=0}^{J} \sum_{k=0}^{K}\left|\overline{b_{i j k}}\right|, 1\right\} .
$$

Let a constant $c_{40} \geqq 1$ and a positive integer $D$ satisfy

$$
\left\{\begin{array}{l}
\mid \overline{A_{\lambda} \mid}\left(|\lambda|=m_{0}\right), \overline{a_{\lambda} \mid}\left(|\lambda| \leqq m_{0}\right), \overline{1 / A_{\lambda_{0}} \mid} \leqq c_{40}, \\
D A_{\lambda}\left(|\lambda|=m_{0}\right), D a_{\lambda}\left(|\lambda| \leqq m_{0}\right), D\left(1 / A_{\lambda_{0}}\right) \text { are algebraic integers. }
\end{array}\right.
$$

Then we can prove (4.3) and (4.4) in the case $n=1$ and $n>1$ respectively, for any $\mu \in S$ with $|\mu|=m_{0}+m(m \geqq 1)$ by induction in $m$.

$$
\left\{\begin{array}{l}
\left|\overline{a_{\mu}}\right| \leqq\left\{M c_{40}\left(m_{0}+1\right)^{J+K}\right\}^{2 m-1}(m !)^{J+K} C_{40}{ }^{m\left(2 m_{0}+1\right)}, \\
D^{2 m-1} D^{m\left(2 m_{0}-1\right)} a_{\mu} \text { is an algebraic integer. }
\end{array}\right.
$$




$$
\left\{\begin{array}{l}
\left|\overline{a_{\mu}}\right| \leqq\left(4 c_{40}^{2}\right)^{\left(m_{0}+2\right)^{n-1} m^{2(n-1)}}\left\{M\left(m_{0}+1\right)^{n(J+K)}\right\}^{2 m-1}(m !)^{n(J+K)} c_{40}{ }^{m\left(2 m_{0}+1\right)} \\
D^{2\left(m_{0}+2\right)^{n-1} m^{2(n-1)} D^{m\left(2 m_{0}+1\right)} a_{\mu} \text { is analgebraic integer. }}
\end{array}\right.
$$

In the case where $f(z)$ is a power series with powers being non-negative integers, (4.3) and (4.4) lead the theorem. Since the proof of (4.3) is easier than the proof of (4.4), we only prove (4.4). We give a number to each element of $\left\{\mu:|\mu|=m+m_{0}\right\}$ as $: \mu_{1}<\mu_{2}<\cdots<\mu_{l(m)}$. Note that

$$
l(m) \leqq\left(m+m_{0}+1\right)^{n-1} \leqq\left(m_{0}+2\right)^{n-1} m^{n-1} .
$$

By Lemma 9, we have

$$
B_{\lambda_{0}+\mu_{i}}(y)=\sum_{2 m_{0}+m \geq|\lambda| \geq m_{0}+m} A_{\lambda_{0}+\mu_{i}-\lambda}(y) y_{\lambda}+C_{\lambda_{0}+\mu_{i}}(y),
$$

where $C_{\lambda_{0}+\mu_{i}}(y)$ is the coefficient of $z^{\lambda_{0}+\mu_{i}}$ in

$$
\sum_{i \in S} \sum_{j=0}^{J} \sum_{k=0}^{K} b_{i j k} z^{i}\left(\sum_{|\lambda|<m_{0}+m} y_{\lambda} z^{\lambda}\right)^{j}\left(\sum_{|\lambda|<m_{0}+m} y_{\lambda} z^{i \Omega}\right)^{k} .
$$

If $\lambda>\mu_{i}$, then $\lambda_{0}+\mu_{i}-\lambda \notin S$ or $\lambda_{0}+\mu_{i}-\lambda<\lambda_{0}$. In any case, $A_{\lambda_{0}+\mu_{i}-\lambda}=0$. Substituting $a_{\lambda}$ to $y_{\lambda}$ in (4.4), we have

$$
A_{\lambda_{0}+\mu_{i}-\mu_{1}} a_{\mu_{1}}+A_{\lambda_{0}+\mu_{i}-\mu_{2}} a_{\mu_{2}}+\cdots+A_{\lambda_{0}} a_{\mu_{i}}=-C_{\lambda_{0}+\mu_{i}},
$$

where $C_{\lambda_{0}+\mu_{i}}$ denotes the values of $C_{\lambda_{0}+\lambda_{i}}(y)$ at $y_{\lambda}=a_{\lambda}(\lambda \in S)$. Therefore

$$
\left(\begin{array}{cccc}
A_{\lambda_{0}} & & & \\
& A_{\lambda_{0}} & & 0 \\
& & \ddots & \\
& * & & A_{\lambda_{0}}
\end{array}\right)\left(\begin{array}{c}
a_{\mu_{1}} \\
a_{\mu_{2}} \\
\vdots \\
a_{\mu_{l(m)}}
\end{array}\right)=\left(\begin{array}{c}
-C_{\lambda_{0}+\mu_{1}} \\
-C_{\lambda_{0}+\mu_{2}} \\
\vdots \\
-C_{\lambda_{0}+\mu_{l(m)}}
\end{array}\right),
$$

where the entries in $*$ consist of $A_{\lambda}\left(|\lambda|=m_{0}\right)$. Hence by (4.2) we have

$$
\begin{aligned}
\left|a_{\mu_{i}}\right| \leqq & c_{40}{ }^{l(m)} l(m) 2^{l(m)} c_{40}{ }^{l(m)} M\left(m_{0}+m\right)^{n(J+K)} \\
& \times \max \left|a_{\lambda_{1}} \cdots a_{\lambda_{r}} a_{\nu_{1}} \cdots a_{\nu_{s}}\right| \\
\leqq & \max \left(4 c_{40}{ }^{2}\right)^{\left(m_{0}+2\right)}{ }^{n-1} m^{n-1} M\left(m_{0}+1\right)^{n(J+K)} m^{n(J+K)} \\
& \times\left|a_{\lambda_{1}} \cdots a_{\lambda_{n}} a_{\nu_{1}} \cdots a_{\nu_{s}}\right|
\end{aligned}
$$

where max is taken over all $\left(\lambda_{1}, \cdots, \lambda_{r}, \nu_{1}, \cdots, \nu_{s}\right)$ such that

$$
\left\{\begin{array}{l}
\lambda_{i}, \nu_{j} \in S,\left|\lambda_{i}\right|=m_{0}+m_{i} \text { with } 1 \leqq m_{i}<m,\left|\nu_{j}\right| \leqq m_{0}, \\
\left(m_{0}+m_{1}\right)+\cdots+\left(m_{0}+m_{r}\right)+\left|\nu_{1}\right|+\cdots+\left|\nu_{s}\right| \leqq m+2 m_{0} .
\end{array}\right.
$$

If $d$ is a common multiple of all $d\left(a_{\lambda_{1}} \cdots a_{\lambda_{r}} a_{\nu_{1}} \cdots a_{\nu_{s}}\right)$ with $\left(\lambda_{1}, \cdots, \lambda_{r}, \nu_{1}, \cdots, \nu_{s}\right)$ satisfying (4.8), then $D^{2 l(m)} d a_{\mu_{i}}$ is an algebraic integer. If $m=1$, then $r=0$, and

$$
\begin{aligned}
& \left|a_{\nu_{1}} \cdots a_{\nu_{s}}\right| \leqq c_{40}{ }^{2 m_{0}+1}, \\
& D^{2 m_{0}+1} a_{\nu_{1}} \cdots a_{\nu_{s}} \text { is an algebraic integer. }
\end{aligned}
$$


This implies (4.4). If $m>1$, then by the induction hypothesis,

$$
\begin{aligned}
& \left|a_{\lambda_{1}} \cdots a_{\lambda_{r}} a_{\nu_{1}} \cdots a_{\nu_{s}}\right| \leqq\left(4 c_{40}\right)^{\left(m_{0}+2\right)^{n-1}\left(m_{1}{ }^{2(n-1)}+\cdots+m_{r} r^{2(n-1)}\right)} \\
& \times\left\{M\left(m_{0}+1\right)^{n(J+K)}\right\}^{2\left(m_{1}+\cdots+m_{r}\right)-r}\left(m_{1} ! \cdots m_{r} !\right)^{n(J+K)} \\
& \times c_{40}{ }^{\left(m_{1}+\cdots+m_{r}\right)\left(2 m_{0}+1\right)} c_{40}{ }^{m+2 m_{0}-\left(m_{0}+m_{1}\right)-\cdots-\left(m_{0}+m_{r}\right)} .
\end{aligned}
$$

If $r=0$, then $\left|\overline{a_{\nu_{1}} \cdots a_{\nu_{s}}}\right| \leqq c_{40}{ }^{m+2 m_{0}}$ and

$$
\begin{aligned}
& \left(4 c_{40}{ }^{2}\right)^{\left(m_{0}+2\right){ }^{n-1} m n-1} M\left(m_{0}+1\right)^{n(J+K)} m^{n(J+K)} \mid \overline{a_{\nu_{1}} \cdots a_{\nu_{s}} \mid} \\
\leqq & \left(4 c_{40}\right)^{\left(m_{0}+2\right) n-1 m^{2(n-1)}}\left\{M\left(m_{0}+1\right)^{n(J+K)}\right\}^{2 m-1}(m !)^{n(J+K)} c_{40}{ }^{m\left(2 m_{0}+1\right)} .
\end{aligned}
$$

If $r=1$, then

$$
\begin{aligned}
& \left(4 c_{40}{ }^{2}\right)^{\left(m_{0}+2\right)}{ }^{n-1} m^{n-1} M\left(m_{0}+1\right)^{n(J+K)} m^{n(J+K)} \mid \overline{a_{\lambda_{1}} a_{\nu_{1}} \cdots a_{\nu_{s}}} \\
& \leqq\left(4 c_{40}^{2}\right)^{\left.\left(m_{0}+2\right) n-1\left(m^{n-1+(m-1)}\right)^{(n-1)}\right)}\left\{M\left(m_{0}+1\right)^{n(J+K)}\right\}^{2(m-1)} \\
& \times(m \times(m-1) !)^{n(J+K)} c_{40}{ }^{m\left(2 m_{0}+1\right)} \\
& \leqq\left(4 c_{40}\right)^{\left(m_{0}+2\right) n-1 m^{2(n-1)}}\left\{M\left(m_{0}+1\right)^{n(J+K)}\right\}^{2 m-1}(m !)^{n(J+K)} c_{40}{ }^{m\left(2 m_{0}+1\right)} \text {, }
\end{aligned}
$$

by the inequality $m^{n-1}+(m-1)^{2(n-1)} \leqq\left(m+(m-1)^{2}\right)^{n-1} \leqq m^{2(n-1)}$. If $r \geqq 2$, then $m_{1}+\cdots+m_{r} \leqq m$ by (4.8), and

$$
\begin{aligned}
& \left(4 c_{40}{ }^{2}\right)^{\left(m_{0}+2\right)^{n-1} m^{n-1}}\left\{M\left(m_{0}+1\right)^{n(J+K)}\right\} m^{n(J+K)}\left|a_{\lambda_{1}} \cdots a_{\lambda_{r}} a_{\nu_{1}} \cdots a_{\nu_{s}}\right| \\
\leqq & \left(4 c_{40}{ }^{2}\right)^{\left(m_{0}+2\right)}{ }^{n-1}\left(m_{1} 2(n-1)+\cdots+m_{r}{ }^{2(n-1)}+m^{n-1)}\right. \\
& \times\left\{M\left(m_{0}+1\right)^{n(J+K)}\right\}^{2 m-1}\left(m \times m_{1} ! \cdots m_{r} !\right)^{n(J+K)} c_{40}{ }^{m\left(2 m_{0}+1\right)} \\
\leqq & \left(4 c_{40}{ }^{2}\right)^{\left.\left(m_{0}+2\right)\right)^{n-1} m^{2(n-1)}}\left\{M\left(m_{0}+1\right)^{n(J+K)}\right\}^{2 m-1}(m !)^{n(J+K)} c_{40} m\left(2 m_{0}+1\right)
\end{aligned}
$$

by the inequalities $m \times m_{1} ! \cdots m_{r} ! \leqq m !$ and

$$
\begin{gathered}
m_{1}{ }^{2(n-1)}+\cdots+m_{r}{ }^{2(n-1)}+m^{r-1} \\
\leqq \\
\left(m_{1}{ }^{2}+\cdots+m_{r}{ }^{2}+m\right)^{r-1} \leqq m^{2(r-1)} .
\end{gathered}
$$

The denominator of $a_{\mu_{i}}$ is also estimated in the same way. These imply (4.4).

In general case, there is a polynomial $P(z, u, v), \overline{\mathbf{Q}}\left[z_{1}, \cdots, z_{n}, u, v\right]$ such that

$$
P_{u}(z, f(z), f(T z))=\Sigma A_{h_{1} \cdots h_{n}} z_{1}^{h_{1}} \cdots z_{n}{ }^{h_{n}} \neq 0 .
$$

$m_{0}^{\prime}$ is the least number in all $h_{1}+\cdots+h_{n}$ with $A_{h_{1} \cdots h_{n}} \neq 0$. Let $a_{\lambda_{1} \cdots \lambda_{n}} \neq 0$ and $\lambda_{i} \leqq h(1 \leqq i \leqq n)$. Substituting $z_{i}{ }^{t \delta_{n h+m_{0}^{\prime}}}$ to $z_{i}$, we can treat $\left\{a_{h_{1} \cdots h_{n}}: h_{1}+\cdots+h_{n}\right.$ $\leqq n h\}$ in the same way as above with $m_{0}=t \delta_{n h+m_{0}^{\prime}} m_{0}^{\prime}\left(\leqq c_{41} h^{\eta}\right)$ and $m_{0}+m=$ $\left(\lambda_{1}+\cdots+\lambda_{n}\right) t \delta_{n h+m_{0}^{\prime}}\left(\leqq c_{42} h^{1+\eta}\right)$. Thus we have the theorem.

Similarly we can prove Theorem 3 , and we omit the proof. 
On a problem of Mahler for transcendency of function values II

\section{References}

[1] Lang, S., Introduction to Transcendental Numbers. Addison-Wesley, 1966.

[2] Loxton, J.H. and van der Poorten, A.J., Transcendence and algebraic independence by a method of Mahler. Transcendence Theory-Advances and Applications ed. Baker, A. and Masser, D. W., Academic Press (1977), 211-226.

[3] Loxton, J.H. and van der Poorten, A. J., Arithmetic properties of certain functions in several variables. Journal of number theory 9 (1977), 87-106.

[4] Mahler, K., Arithmetische Eigenschaften der Lösugen einer Klasse von Funktionalgleichungen. Math. Ann. 101 (1929), 342-366.

[5] Mahler, K., Über das Verschwinden von Potenzreihen mehrerer Veränderlichen in speziellen Punktfolgen. Math. Ann. 103 (1930), 573-587.

[6] Mahler, K., Arithmetische Eigenschaften einer Klass transzendental-transzendenter funktionen. Math. Z. 32 (1930), 545-585.

[7] Mahler, K., Remarks on a paper by W. Shwarz. Journal of number theory 1 (1969), 512-521.

[8] Masser, D.W., A vanishing theorem for power series. Invent. math. 67 (1982), 275-296.

[9] Nishioka, K., On a problem of Mahler for transcendency of function values. J. Austral. Math. Soc. (Series A) 33 (1982), 386-393.

Department of Mathematics

Nara Women's University

Kita-Uoya Nishimachi, Nara

630 Japan 